\title{
High protein intake in neonatal period induces glomerular hypertrophy and sclerosis in adulthood in rats born with IUGR
}

\author{
Farid Boubred' ${ }^{1}$ Eloïse Delamaire ${ }^{2}$, Christophe Buffat ${ }^{3}$, Laurent Daniel ${ }^{4}$, Clair-Yves Boquien², Dominique Darmaun ${ }^{2}$ and \\ Umberto Simeoni ${ }^{5}$
}

\begin{abstract}
BACKGROUND: Intrauterine growth restriction (IUGR) and postnatal nutrition are risk factors for cardiovascular and renal diseases in both humans and animals. The long-term renal effects of protein intake early in life remain unknown. The objective was to evaluate the effects of a neonatal feeding with high protein (HP) milk on renal functions and structure in IUGR male rats.
\end{abstract}

METHODS: Maternal gestational low protein diet was used to produce IUGR. At day 5, IUGR pups were gastrostomized in the "pup-in-the cup" model and received either normal protein (NP) milk or HP (+50\% protein content) milk until day 21. After weaning, the animals were fed the same standard diet. Renal functions and structure were assessed at postnatal day 18 (D18) and in adult offspring.

RESULTS: During the preweaning period, the postnatal weight gain between the two groups was unaffected. On D18, kidneys from HP offspring were heavier with significant glomerular hypertrophy $(+40 \%, P<0.05)$. HP diet was associated with significant proteinuria and glomerulosclerosis $(+49 \%$, $P<0.05)$. Glomerular number was unaltered.

CONCLUSION: Neonatal HP feeding following IUGR affects renal functions and structure at adulthood. These alterations may result from a single nephron glomerular hyperfiltration.

ow birth weight, especially related to intrauterine growth -restriction (IUGR), is a risk factor for cardiovascular and chronic kidney disease (CKD) in adulthood in both humans and animals (1-4). While early postnatal catch-up growth or postnatal overnutrition have long been known to play a role in the development of cardiovascular diseases in adults born with a low birth weight, the effect of early growth and nutrition on CKD remain unknown in humans (5-8).

The pathophysiological mechanism is complex and may involve a reduction in nephron number $(3,9-12)$. As proposed by Brenner et al. $(9,10)$, in IUGR offspring with reduced nephron number, in order to meet excretory demand, an adaptive single nephron glomerular hyperfiltration (SNGHF) occurs with glomerular hypertrophy and tubular enlargement. The subsequent glomerular hypertension (HT) leads, in the long run, to renal injury and CKD with proteinuria, glomerular sclerosis, impaired glomerular filtration rate, and HT. In experimental studies, early postnatal overfeeding has been demonstrated to enhance this adaptive glomerular mechanism and to accelerate the development of HT and CKD (8,13-15).

In low-birth-weight infants, it is recommended to provide a high protein diet in order to limit postnatal growth restriction and to prevent neonatal morbidity and long-term neurocognitive impairment; however, the long-lasting impact of such a nutritional strategy on the kidney has been insufficiently studied (16). In adults, high protein intake is known to induce renal hypertrophy and glomerular sclerosis through a SNGHF, especially when the nephron number is reduced (17-19). In young animals, especially in IUGR rat offspring, we and others have shown that early postnatal overnutrition induced obesity, cardiovascular, metabolic, and renal diseases at adulthood (13-15). Nevertheless, these experimental models cannot accurately determine the effects of neonatal protein intake per se. Using the "pup-in-the cup" model, we aimed to demonstrate in IUGR pups (obtained by maternal low protein diet) that a high protein intake $(+50 \%)$ limited to the preweaning period was sufficient to induce early renal hypertrophy and long-term glomerular sclerosis once pups reached adulthood.

\section{RESULTS}

Some of the results regarding growth and metabolic functions have been published elsewhere (20).

\section{Body Weight and Growth}

Birth weight and growth up to the weaning (D21) of IUGR rat pups exposed to normal protein (NP) formula and high protein (HP) formula did not differ (20). However, on D160, HP offspring were significantly heavier than NP offspring, even though both groups received the same chow diet from the time of weaning ( $585 \pm 81$ vs. $517 \pm 49 \mathrm{~g}$, in HP and NP offspring, respectively, $P<0.01)$.

\footnotetext{
'Equipe d'accueil 2193, Division of Neonatology, Faculté de Médecine, Aix-Marseille Université, Marseille, France; ${ }^{2}$ Institut national de la recherche agronomique, Université de Nantes UMR 1280, Physiologie des Adaptations Nutritionnelles, Nantes, France; ${ }^{3}$ Laboratoire de biologie moléculaire, Assistance publique hôpitaix de Marseille, Aix-Marseille Université, Marseille, France; ${ }^{4}$ Equipe d'accueil 3281, Faculté de Médecine, Aix-Marseille Université, Marseille, France; ${ }^{5}$ Developmental origins of health and disease Laboratory, Université de Lausanne, Switzerland. Correspondence: Farid Boubred (farid.boubred@ap-hm.fr)

Received 28 March 2015; accepted 22 June 2015; advance online publication 14 October 2015. doi:10.1038/pr.2015.176
} 


\section{Renal Structure in D18 Pups}

While body weights were not different, kidneys were significantly heavier in HP pups than that in NP pups $(0.24 \pm 0.02$ vs. $0.19 \pm 0.02 \mathrm{mg}, P<0.05)$. This difference was not related to higher nephron number, as this number was similar in both groups (Figure 1a). Differences in kidney weights resulted from glomerular hypertrophy, as demonstrated by the significant increase in mean glomerular volume $(\mathrm{GV})$ in HP pups $(+49 \%, P<0.01$; Figure 1b)

\section{Renal Function and Structure in Adult Offspring}

On D40 and D160, creatinine clearance (ClCreat) did not differ between groups (Figure 2a); however, proteinuria was significantly elevated in HP offspring from D40 $(P<0.05$; Figure $2 b)$.
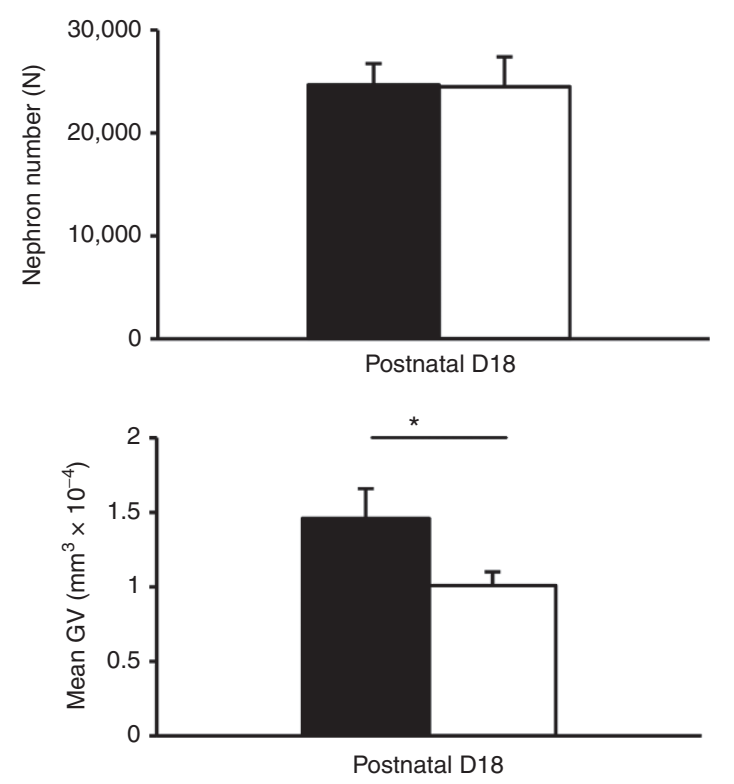

Figure 1. (a,b) Neonatal high protein diet induces renal hypertrophy and fails to improve nephron endowment in 18-d-old pups. Nephron number in IUGR offspring exposed to high (HP, dark bar) and normal (NP, open bar) protein diet were similar. Mean glomerular volume (MGV) in HP offspring (dark bar) was higher than NP offspring (open bar) ( $n=7$ in each group; one-way ANOVA $* P<0.01$ ).
Moreover, the structure of their kidney was more affected with significant glomerular sclerosis $(+47 \%, P=0.002$; Figure $2 \mathrm{c})$. Nephron number was similar between groups $(29,620 \pm 3,470$ vs. $28,933 \pm 2,800$ in $\mathrm{HP}$ and NP offspring, respectively, $P=$ $0.81)$.

\section{DISCUSSION}

To our knowledge, this is the first study that specifically investigated the long-term renal impact of neonatal HP intake in rat pups. Using the neonatal gastrostomy (pup-in-the cup) model, we showed that feeding IUGR male rat offspring with neonatal $\mathrm{HP}$ milk induced renal hypertrophy as early as the neonatal period and proteinuria and glomerular sclerosis at adulthood. These findings may result from a SNGHF.

In this study, we used the gastrostomy with pup-in-the cup model to assess specifically the effects of a neonatal HP diet on kidney functions and structure. This model allows manipulations and investigations of a specific nutrient for a limited period (21). Here, we investigated the specific effects of a HP diet limited to the neonatal period, as after weaning all animals were fed the same standard rat chow ad libitum. Various models have been developed in order to evaluate the longterm consequences of early nutrition (13-15). We previously showed that early neonatal overfeeding, obtained by reduction of litter size down to three pups per litter, accelerated the development of HT and renal disease, especially in IUGR offspring (13). Nevertheless, in such a model, overfeeding provides global high caloric sodium and protein intakes and fails to distinguish the role of each nutrient. In another study, Chen el al. (22) reported impaired renal function and glomerular sclerosis in IUGR adult offspring exposed to a postnatal HP diet. In that study, pups were exposed to a lactating mother being fed a HP diet and were weaned on the same HP diet until the end of experiments at adulthood. However, the design of the study does not allow to delineate the specific effect of neonatal protein intake: since (i) feeding nursing mothers an HP diet does not necessarily translate into a higher protein content in milk and (ii) the adverse renal effects observed at adulthood could be attributed to the prolonged exposure to the HP diet after a

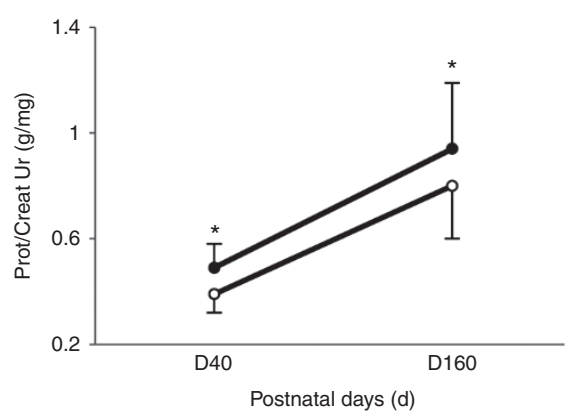

b

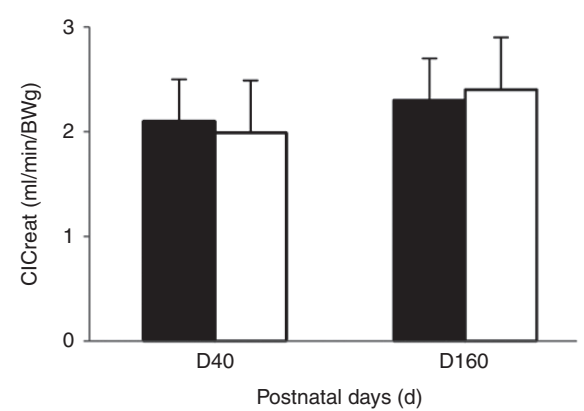

C

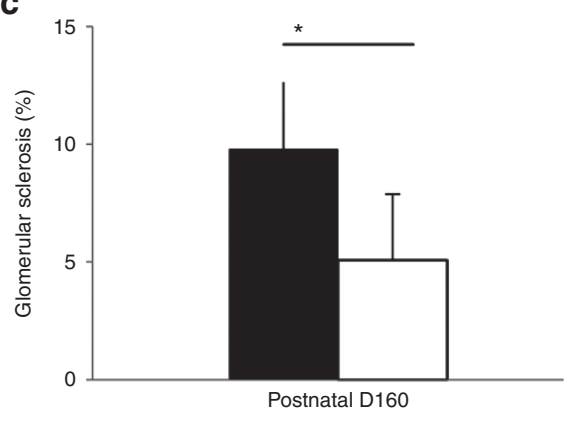

Figure 2. Neonatal high protein (HP) diet induces proteinuria and glomerular sclerosis at adulthood ( $n=7 /$ group). (a) Proteinuria as assessed by urinary protein/creatinine ratio (Prot/Creat) was more elevated in HP offspring (dark circle) than NP offspring (open circle) from postnatal day $40\left(^{*} P<0.05\right.$ ). (b) Glomerular filtration rate evaluated by clearance of creatinine (ClCreat) was similar in both groups (HP, dark bar; NP, open bar). (c) In 160-day-old offspring, glomerular sclerosis using the percentage of total glomerular surface area colored by the Sirius red, a marker of collagen accumulation, was $49 \%$ higher in HP offspring (dark bar) than that in NP offspring (open bar). ${ }^{*} P<0.01$. 


\section{Articles $\mid$ Boubred et al.}

weaning until adulthood. In contrast, our study was specifically designed to investigate the renal effects of a neonatal HP intake which was provided directly to the pups during the neonatal period through manipulation of milk protein content.

HP formula used in this study contained more calories than NP formula. We used such formula to closely mimic the enriched formula used routinely for low-birth-weight infants with higher protein and overall energy contents than standard formula. The renal effects observed in HP offspring may however result from HP content. Indeed, difference in calories between both formula was primarily attributable to HP content: HP formula supplied a moderate $11 \%$ increase in calories (172 vs. $155 \mathrm{kcal} / 100 \mathrm{~g}$ ) and $50 \%$ more protein than NP formula.

We observed that the neonatal HP diet failed to restore nephron numbers but induced renal hypertrophy. The lack of effect on nephron endowment may result from a relative resistance of the IUGR kidney to postnatal nutrition and the end stage of nephrogenesis at which pups were exposed to HP diet. In rodents, in which nephrogenesis begins at midgestation and continues after birth up to days $7-10$, neonatal environment, especially nutrition, can influence nephron endowment $(23,24)$. As compared with normal birth weight (NBW) offspring, early neonatal overfeeding fails to improve postnatal nephrogensis in IUGR pups; this is due in part to impaired development of nephron progenitors cells $(13,25)$. Also, higher kidney weights in HP offspring are more likely to result from renal hypertrophy. Renal hypertrophy may be related to the SNGHF induced by renal filtered overload of amino acids. High amino-acid concentrations in proximal tubule stimulate tubular reabsorption, including sodium reabsorption. The consequent low sodium concentrations in distal tubule inhibit tubuloglomerular feedback responsible for passive vasodilatation of the afferent glomerular arterioles and SNGHF. The functional glomerular and tubular changes are accompanied by glomerular dilatation and tubular enlargement, which are responsible for renal hypertrophy $(10,17,18,26)$.

It remains unknown whether such SNGHF with renal hypertrophy occurring during the neonatal period is sufficient by itself to induce proteinuria and glomerular sclerosis later on at adulthood after stopping HP intake. As proposed by Brenner et al., and suggested by our recent work, to meet excretory demands due to HP diet, a SNGHF occurs associated with glomerular HT and changes in renal structure $(10,13,17)$. In the long run, a vicious cycle takes place, resulting in renal injury, proteinuria, glomerular sclerosis, impaired renal function and thus HT (defining CKD). According to this hypothesis, this progression of renal disease is the consequence of renal injury initiated during the neonatal period. However, additional factors linked in part to the neonatal HP diet have to be considered. Indeed, neonatal HP diet has been demonstrated to accelerate postnatal weight gain with mesenteric fat mass accretion, hypertriglyceridemia, hyperinsulinism, and resistance to leptin $(20,27)$. These changes are known to increase SNGFR, to affect the glomerular endothelium barrier, to impair endothelium-dependent vasodilatation, and to stimulate the sympathetic nervous activity responsible for HT, one of the leading causes of CKD (28-32). Even though blood pressure was not monitored in the current study, we hypothesize that such metabolic, hormonal, and vascular changes enhanced the adaptive glomerular hemodynamic changes and renal injury already acquired during the neonatal period and accelerated the occurrence of proteinuria and glomerular sclerosis (33). Further investigation would be needed to confirm this hypothesis.

In this study, we chose to investigate the effects of a neonatal HP diet in IUGR male offspring. This study followed on our previous work in which we demonstrated that IUGR neonatally overfed male offspring were particularly susceptible to vascular and renal diseases as compared to IUGR female and NBW offspring (13). Our study was not designed to evaluate the effect of HP diet in NBW offspring. Another study, using a similar model of artificial feeding, showed unchanged kidney weight in NBW pups fed milk with 30\% increase in protein content (34). Renal function and structure, however, were not investigated in that study.

\section{Clinical Implications}

Extrapolation from this study to human IUGR should be made with caution since current findings were observed in a specific experimental model. The model used likely is relevant for the IUGR observed in the developing world, in which more than 30 million infants are believed to be born with IUGR yearly as a consequence of maternal undernutrition (35). In the industrialized world, alterations in placental blood flow and preeclampsia are leading causes of IUGR so that IUGR due to maternal protein diet restriction may not be relevant. Recent studies, however, suggest that (i) both settings share some striking similarities, since impaired maternal-fetal amino-acid transfer has been observed in human IUGR of vascular origin and (ii) impairment in the placental transport of amino acids has been found precede growth alterations in a rat model of IUGR induced by protein diet restriction (36-38), implying decreased amino acid transport may be a cause, rather than a consequence, of IUGR in that model. Such evidence therefore argues for the potential relevance of the experimental model used in this study for human IUGR. Considering such caution in transposing findings to humans, our findings highlight the long-term renal consequences of neonatal HP diet in LBW infants. While no study has clearly explored the renal effects of a neonatal HP diet in these infants, two recent studies reported renal hypertrophy $(+10 \%$ to $+25 \%$ increase in kidney volume) in 3- and 6-mo-old NBW infants exposed from birth to HP formulas $(39,40)$. The long-term consequences are unknown.

In conclusion, in IUGR rat pups, a HP intake, limited to the neonatal period, is associated with renal hypertrophy, proteinuria, and glomerular sclerosis at adulthood. This association may result from a single nephron glomerular hyperfiltration. Our findings highlight the needed renal follow-up of lowbirth-weight infants exposed to HP diet early in life. Further studies are warranted to clearly investigate the short- and longterm renal impact of a neonatal HP diet in LBW infants and to evaluate early markers of renal dysfunctions. 


\section{METHODS}

\section{Animals and Diet}

All procedures on animals were performed in accordance with the European Communities Council Directive of 24 November 1986 (86/609/EEC) and approved by the institutional animal care of Nantes University. Eight-week-old male and virgin female Sprague-Dawley rats (Janvier, Le Genest Saint Isle, France) were housed individually and were kept on standard laboratory rat chow diet $2 \mathrm{wk}$ before mating. During experiments, rats had free access to food and tap water ad libitum in a room at 12:12-h light/dark cycle, at controlled temperature $\left(22^{\circ} \mathrm{C}\right)$ and with a constant humidity.

After copulation and when sperm was seen in a vaginal smear, 12 pregnant rats were housed individually and fed a low protein diet $(8 \%$ protein; Arie Block BV, Woerden, The Netherlands). After birth, the weights of all pups were recorded, and the litters were culled to eight pups per litter. As our findings from previous works and evidence from the literature suggest that the long-term effects of early postnatal nutrition affect more males than females, we chose to include only male offspring in this study (13). The pup-in-the cup model with gastrostomy was used to evaluate the effects of a neonatal protein diet on renal functions and structure. The detailed experiments are described elsewhere (20). Briefly, on D5, an intragastric cannula was implanted percutaneously under general anesthesia. Pups were then accommodated in individually warmed cups maintained at 37 ${ }^{\circ} \mathrm{C}$ in a water bath. After a 2-day acclimation period, they were randomly allocated to either a NP diet $(n=14)$ or a HP diet $(n=14)$. The HP diet contained salt $137 \mathrm{mg} / 100 \mathrm{ml}$, proteins $13.1 \mathrm{~g} / 100 \mathrm{ml}$, lipids $12.1 \mathrm{~g} / 100 \mathrm{ml}$, and lactose $3.5 \mathrm{~g} / 100 \mathrm{ml}$ with total energy of $172.6 \mathrm{Kcal} / 100 \mathrm{~g}$ vs. $120 \mathrm{mg} / 100 \mathrm{ml}, 8.7 \mathrm{~g} / 100 \mathrm{ml}, 11.9 \mathrm{~g} / 100 \mathrm{ml}$, and $3.3 \mathrm{~g} / 100 \mathrm{ml}$ with $155 \mathrm{Kcal} / 100 \mathrm{~g}$, respectively, in NP diet. The milk was prepared from a powdered rat milk substitute (Wombaroo Food Products, Glen Osmond, Australia), and protein with free amino acids (Protilight IP3 and Protarmor 800Li; Armor Protéines, SaintBrice-en-Coglès, France) was added to reach a $50 \%$ increase in protein intakes. Pups were then weighted daily to adjust the formula volume delivered through programmable syringe pumps. On day 18 , seven pups from each group were anesthetized and sacrificed for kidney analysis; the remaining pups were weaned on standard laboratory rat chow (on D21) and housed by four in cages. The remaining offspring were monitored for renal function and structure (see below). Noninvasive plethysmography method was used to measure blood pressure in adult offspring; but all measurements were inadequate, out of expected range usually observed in adult rats and unusable unfortunately. We decided not to consider the data.

\section{Determination of Renal Function and Urinary Protein Excretion}

Endogenous creatinine clearance $(\mathrm{CrCl})$ and urinary protein/creatinine ratio (UPr/UCr) were determined in D40 and D160 adult offspring as $\mathrm{CrCl}=\mathrm{UCr} \times \mathrm{V} \times \mathrm{PCr}^{-1}$, where $\mathrm{UCr}$ and $\mathrm{PCr}$ are urinary and plasma creatinine concentrations, respectively, UPr is urinary protein concentration, and $V$ is urinary output. The animals were housed individually for $48 \mathrm{~h}$ in metabolic cages, and 24-hour urine collection was then performed gravimetrically along with a blood sample $(0.5 \mathrm{ml})$. The blood samples were then transferred to heparinized tubes and centrifuged at 3,000 revolutions/min for $15 \mathrm{~min}$ at $4{ }^{\circ} \mathrm{C}$. Plasma creatinine, urinary creatinine, and protein concentrations were measured by a standard autoanalyzer (Synchron LX20 autoanalyzer; Beckman Coulte, Brea, CA). The Jaffé method was used to determine the plasma creatinine concentration.

\section{Morphometric Measurements and Estimation of Nephron Number, GV, and Glomeruloscleosis}

Renal structure was assessed on day 18, at the end of the suckljing period, and on day 160 when the animals were killed with $\mathrm{CO}_{2}$ inhalation (D160). The left kidney was rapidly harvested, weighed, and decapsulated for glomerular counting, and the right kidney was kept for histology analysis.

The number of glomeruli per kidney was determined in the two groups of rats on D18 and D160, as previously described (23). Whole kidneys were briefly incubated in a $50 \%$ hydrochloric acid solution for 30 to $45 \mathrm{~min}$ at $37^{\circ} \mathrm{C}$, the incubation time being dependent on kidney weight. Kidneys were rinsed with tap water and stored overnight at $4{ }^{\circ} \mathrm{C}$ in a gauged flask. Following mechanical dissociation, tubules and glomeruli were suspended in water. Three $0.5 \mathrm{ml}$ aliquots were taken and placed in a hemocytometer-like chamber, and the glomeruli were counted under microscope by three investigators who were unaware of the specimen origin. The three results were averaged, and then, the value was used to determine the total number of glomeruli in the sample and therefore the kidney.

The renal histology and corresponding parameters were analyzed by one investigator (L.D.) who had no prior knowledge of the group to which the rats belonged. One half of the right kidney was fixed in $4 \%$ buffered formaldehyde. The kidneys were then dehydrated through graded alcohols and embedded in paraffin. Transverse sections through the central portion of each kidney and 4- $\mu$ m-thick sections stained with hematoxylin and eosin were obtained for light microscopic examination. In every single section of kidney, all glomeruli (i.e., superficial and juxtamedullary), sectioned through the hilum, were counted and assessed for GV. In each specimen, more than 50 glomerular cross-sections not crossing the outline of the examined field were analyzed for each group, without extensive structure alterations. The profile of a glomerulus was captured and the perimeter of Bowman's capsule was traced using a tablet cursor to determine GV. Cross-sectional tuft area $\left(G_{A}\right)$ was calculated for each glomerulus with a visible vascular pole using image analyzing software (SAMBA 2005 Alcatel; TITN Answare, Rennes, France). GV was then calculated, assuming the glomerulus to be spherical by applying the following mathematical equation as $G V=\beta / k \times(G)^{3 / 2}$, where $\beta$ is the shape coefficient for a sphere $(=1.38)$ and $\mathrm{k}$ is the size distribution coefficient $(=1.1)(47,54)$. GV was determined in 18 - and 160 -d-old offspring.

Glomerular sclerosis was evaluated using Sirius red coloration to visualize fibrillar collagen in 160-d-old offspring. The percentage of glomerular surface area stained with Sirius red was determined. A quantitative analysis was performed by a single examiner (L.D.) using the same colorimetric and light thresholds (NCSS 2004 software, Kaysville, UT). Color threshold was then applied to identify the redstained structure. The results were reported as the mean ratio of Sirius red-stained areas to total glomerular capillary areas.

\section{Statistical Analysis}

Data are presented as means \pm SD. One-way ANOVA with a StudentNewman-Keuls comparison test post hoc analysis (Statview version 5.0 Software; Abacus Concepts, Berkeley, CA) was used to analyze differences between groups. Statistical significance was defined as $P<0.05$.

\section{ACKNOWLEDGMENTS}

We express our gratitude to Wombaroo Food Products (Glen Osmond, Australia) and Armor Protéines (Saint-Brice-en-Coglès, France) for the generous gift of the rat milk substitute and protein supplements, respectively, to G. Poupeau for his surgical assistance, and to the scientists and research technicians at PhAN Research Laboratory, who generously dedicated their time to the pups'"nursing care."

\section{STATEMENT OF FINANCIAL SUPPORT}

This work was supported in part by a grant from the Agence Nationale de la Recherche (French National Agency for Research, ProtNeonat ANR grant \# 05-PNRA-009, Paris, France).

Disclosures: Authors have no conflict of interest.

\section{REFERENCES}

1. Barker DJ. Adult consequences of fetal growth restriction. Clin Obstet Gynecol 2006;49:270-83.

2. White SL, Perkovic V, Cass A, et al. Is low birth weight an antecedent of CKD in later life? A systematic review of observational studies. Am J Kidney Dis 2009;54:248-61.

3. Vehaskari VM, Woods LL. Prenatal programming of hypertension: lessons from experimental models. J Am Soc Nephrol 2005;16:2545-56.

4. López-Bermejo A, Sitjar C, Cabacas A, et al. Prenatal programming of renal function: the estimated glomerular filtration rate is influenced by size at birth in apparently healthy children. Pediatr Res 2008;64:97-9. 


\section{Articles Boubred et al.}

5. Barker DJ, Osmond C, Forsén TJ, Kajantie E, Eriksson JG. Trajectories of growth among children who have coronary events as adults. N Engl J Med 2005;353:1802-9.

6. Adair LS, Cole TJ. Rapid child growth raises blood pressure in adolescent boys who were thin at birth. Hypertension 2003;41:451-6.

7. Huxley RR, Shiell AW, Law CM. The role of size at birth and postnatal catch-up growth in determining systolic blood pressure: a systematic review of the literature. J Hypertens 2000;18:815-31.

8. Simeoni U, Ligi I, Buffat C, Boubred F. Adverse consequences of accelerated neonatal growth: cardiovascular and renal issues. Pediatr Nephrol 2011;26:493-508

9. Brenner BM, Chertow GM. Congenital oligonephropathy and the etiology of adult hypertension and progressive renal injury. Am J Kidney Dis 1994;23:171-5.

10. Brenner BM, Garcia DL, Anderson S. Glomeruli and blood pressure. Less of one, more the other? Am J Hypertens 1988;1(4 Pt 1):335-47.

11. Lelièvre-Pégorier M, Merlet-Bénichou C. The number of nephrons in the mammalian kidney: environmental influences play a determining role. Exp Nephrol 2000;8:63-5.

12. Hinchliffe SA, Lynch MR, Sargent PH, Howard CV, Van Velzen D. The effect of intrauterine growth retardation on the development of renal nephrons. Br J Obstet Gynaecol 1992;99:296-301.

13. Boubred F, Daniel L, Buffat C, et al. Early postnatal overfeeding induces early chronic renal dysfunction in adult male rats. Am J Physiol Renal Physiol 2009;297:F943-51.

14. Luyckx VA, Compston CA, Simmen T, Mueller TF. Accelerated senescence in kidneys of low-birth-weight rats after catch-up growth. Am J Physiol Renal Physiol 2009;297:F1697-705.

15. Vickers MH, Breier BH, Cutfield WS, Hofman PL, Gluckman PD. Fetal origins of hyperphagia, obesity, and hypertension and postnatal amplification by hypercaloric nutrition. Am J Physiol Endocrinol Metab 2000;279:E83-7.

16. Agostoni C, Buonocore G, Carnielli VP, et al.; ESPGHAN Committee on Nutrition. Enteral nutrient supply for preterm infants: commentary from the European Society of Paediatric Gastroenterology, Hepatology and Nutrition Committee on Nutrition. J Pediatr Gastroenterol Nutr 2010;50:85-91.

17. Brenner BM, Meyer TW, Hostetter TH. Dietary protein intake and the progressive nature of kidney disease: the role of hemodynamically mediated glomerular injury in the pathogenesis of progressive glomerular sclerosis in aging, renal ablation, and intrinsic renal disease. $\mathrm{N}$ Engl J Med 1982;307:652-9.

18. Slomowitz LA, Gabbai FB, Khang SJ, et al. Protein intake regulates the vasodilatory function of the kidney and NMDA receptor expression. Am J Physiol Regul Integr Comp Physiol 2004;287:R1184-9.

19. Knight EL, Stampfer MJ, Hankinson SE, Spiegelman D, Curhan GC. The impact of protein intake on renal function decline in women with normal renal function or mild renal insufficiency. Ann Intern Med 2003;138:460-7.

20. Delamaire E, Parnet P, Coupé B, et al. Long term metabolic impact of high protein neonatal feeding: a preliminary study in male rat pups born with a low birth weight. Clin Nutr 2012;31:741-8.

21. Patel MS, Vadlamudi SP, Johanning GL. Overview of pup in a cup model: hepatic lipogenesis in rats artificially reared on a high-carbohydrate formula. J Nutr 1993;123:Suppl 2:373-7.

22. Chen J, Xu H, Shen Q, Guo W, Sun L. Effect of postnatal high-protein diet on kidney function of rats exposed to intrauterine protein restriction. Pediatr Res 2010;68:100-4.
23. Boubred F, Buffat C, Feuerstein JM, et al. Effects of early postnatal hypernutrition on nephron number and long-term renal function and structure in rats. Am J Physiol Renal Physiol 2007;293:F1944-9.

24. Schreuder MF, Nyengaard JR, Remmers F, van Wijk JA, Delemarrevan de Waal HA. Postnatal food restriction in the rat as a model for a low nephron endowment. Am J Physiol Renal Physiol 2006;291:F1104-7.

25. Welham SJ, Wade A, Woolf AS. Protein restriction in pregnancy is associated with increased apoptosis of mesenchymal cells at the start of rat metanephrogenesis. Kidney Int 2002;61:1231-42.

26. Schrijvers BF, Rasch R, Tilton RG, Flyvbjerg A. High protein-induced glomerular hypertrophy is vascular endothelial growth factor-dependent. Kidney Int 2002;61:1600-4.

27. Coupé B, Delamaire E, Hoebler C, et al. Hypothalamus integrity and appetite regulation in low birth weight rats reared artificially on a high-protein milk formula. J Nutr Biochem 2011;22:956-63.

28. Griffin KA, Kramer H, Bidani AK. Adverse renal consequences of obesity. Am J Physiol Renal Physiol 2008;294:F685-96.

29. Lim K, Lombardo P, Schneider-Kolsky M, Hilliard L, Denton KM, Black MJ. Induction of hyperglycemia in adult intrauterine growth-restricted rats: effects on renal function. Am J Physiol Renal Physiol 2011;301: F288-94.

30. Yamamoto Y, Maeshima Y, Kitayama H, et al. Tumstatin peptide, an inhibitor of angiogenesis, prevents glomerular hypertrophy in the early stage of diabetic nephropathy. Diabetes 2004;53:1831-40.

31. Shinozaki K, Kashiwagi A, Masada M, Okamura T. Molecular mechanisms of impaired endothelial function associated with insulin resistance. Curr Drug Targets Cardiovasc Haematol Disord 2004;4:1-11.

32. Montanaro MS, Allen AM, Oldfield BJ. Structural and functional evidence supporting a role for leptin in central neural pathways influencing blood pressure in rats. Exp Physiol 2005;90:689-96.

33. Boubred F, Saint-Faust M, Buffat C, Ligi I, Grandvuillemin I, Simeoni U. Developmental origins of chronic renal disease: an integrative hypothesis. Int J Nephrol 2013;2013:346067.

34. des Robert C, Li N, Caicedo R, et al. Metabolic effects of different protein intakes after short term undernutrition in artificially reared infant rats. Early Hum Dev 2009;85:41-9.

35. Bernstein IM, Horbar JD, Badger GJ, Ohlsson A, Golan A. Morbidity and mortality among very-low-birth-weight neonates with intrauterine growth restriction. The Vermont Oxford Network. Am J Obstet Gynecol 2000;182(1 Pt 1):198-206.

36. Marconi AM, Paolini CL, Stramare L, et al. Steady state maternal-fetal leucine enrichments in normal and intrauterine growth-restricted pregnancies. Pediatr Res 1999;46:114-9.

37. Paolini CL, Marconi AM, Ronzoni S, et al. Placental transport of leucine, phenylalanine, glycine, and proline in intrauterine growth-restricted pregnancies. J Clin Endocrinol Metab 2001;86:5427-32.

38. Jansson N, Pettersson J, Haafiz A, et al. Down-regulation of placental transport of amino acids precedes the development of intrauterine growth restriction in rats fed a low protein diet. J Physiol 2006;576(Pt 3): 935-46.

39. Schmidt IM, Damgaard IN, Boisen KA, et al. Increased kidney growth in formula-fed versus breast-fed healthy infants. Pediatr Nephrol 2004;19:1137-44.

40. Escribano J, Luque V, Ferre N, et al. Increased protein intake augments kidney volume and function in healthy infants. Kidney Int 2011;79: 783-90. 